\title{
Natalizumab administration in multiple sclerosis patients during active SARS-CoV-2 infection: a case series
}

\author{
Clara G. Chisari ${ }^{1 \dagger}$, Simona Toscano ${ }^{1 \dagger}$, Sebastiano Arena ${ }^{1}$, Chiara Finocchiaro ${ }^{1}$, Arturo Montineri ${ }^{2}$ and \\ Francesco Patti ${ }^{1 *}$ (1)
}

\begin{abstract}
Background: The Coronavirus disease 2019 (COVID-19) caused by the new Severe Acute Respiratory Syndrome Coronavirus 2 (SARS-CoV-2) has become a pandemic, affecting the therapeutic management for Multiple Sclerosis (MS). Any decision regarding the discontinuation of high-potency agents for moderate and highly active MS should be carefully evaluated, taking into account the potential risk of rebound of the disease. In particular, no data about clinical outcome of patients with MS receiving Natalizumab (NTZ) during active COVID-19 infection have been reported yet.
\end{abstract}

Cases presentation: We reported on 6 patients treated with NTZ for relapsing MS during active COVID-19 infection, who recovered without reporting any worsening or new symptoms. Most of the patients were asymptomatic, with the exception of one patient who had a slight worst COVID-19 clinical course. No patients received O2-therapy or required intensive care. No neurological complications were observed.

Conclusions: This paper reported the clinical outcome of patients with MS receiving NTZ during active COVID-19 infection. This case series suggests that treatment with NTZ during pandemic is relatively safe and might be continued in selected patients who are infected by COVID-19, thereby reducing the risk of MS disease rebound.

Keywords: Multiple sclerosis, COVID-19, SARS-CoV-2 active infection, Natalizumab

\section{Background}

During the last year, the new Coronavirus Disease 19 (COVID-19) caused by the new Severe Acute Respiratory Syndrome Coronavirus 2 (SARS-CoV-2) has become a pandemic, severely affecting world's entire population [1]. Older age, male sex, obesity, and presence of comorbidities have been associated to higher risk of mortality due to COVID-19 [2].

\footnotetext{
*Correspondence: patti@unict.it

${ }^{\dagger}$ Clara G. Chisari and Simona Toscano contributed equally to this work.

${ }^{1}$ Department of Medical and Surgical Sciences and Advanced

Technologies "G.F. Ingrassia", section of Neurosciences, University

of Catania, via Santa Sofia 78, 95123 Catania, Italy

Full list of author information is available at the end of the article
}

People with Multiple Sclerosis (MS) were immediately identified as vulnerable subjects and, more specifically, older age, worse disability, progressive course of MS, comorbidities and male sex were considered as risk factors for more severe COVID-19 course [3-5]. However, although MS patients typically have a higher infection risk due to both the autoimmune pathogenesis of the disease and the frequent use of immunosuppressant drugs, an acceptable level of safety during COVID-19 pandemic has been reported for most of the disease-modifying therapies (DMTs) [6]. Indeed, several studies have suggested that injectables DMTs have a favorable immune profile without an increased exposure risk, while concerns have been raised about the use of oral or 
intravenous high-potency agents for moderate and highly active MS [6, 7]. Particularly, more recent data have indicated an increased risk of serious infections for patients under B cell-depleting therapies [6].

Despite that, any decision regarding the discontinuation of highly effective DMTs for MS has to carefully take into account the potential risk of disease rebound. For this reason, European Academy of Neurology (EAN), Associazione Italiana Sclerosi Multipla (AISM) and the Association of British Neurologists (ABN) recommended to hold injectable and oral therapies and delay celldepleting DMTs [8-12]. Moreover, immune-modulating therapies such as the dimethyl-fumarate, teriflunomide, and natalizumab (NTZ) are preferred over cell-depleting drugs during the COVID-19 pandemic for the safer immune profile $[11,12]$. Thanks to its non-cell-depleting mechanism of action, it has been supposed that NTZ unlikely increases COVID-19 infection susceptibility and, thus, is likely safe to be administered in patients already treated and also in naïve patients as a new treatment [11, 13]. In addition, data about NTZ infectious side-effect profile have shown a mild increase in the susceptibility to respiratory viral infections [14].

To date, only two cases of COVID-19 in patients with MS treated with NTZ have been reported, both with mild clinical courses and complete recovery $[15,16]$. In one of them, the use of the extended interval dosing strategy of 6-8 weeks (already described for lowering the risk of Progressive Multifocal Leukoencephalopathy [PML]) has been proposed for decreasing the infection risk and the access to hospital services during the pandemic $[16,17]$.

To date, it has been recommended to continue all injectable and oral DMTs and to consider the administration of NTZ in COVID-19 ward for patients infected by COVID-19, in order to avoid a disease rebound [10-12]. Nevertheless, there are still no data about the effects of continuing NTZ treatment during active COVID-19 infection in MS patients.

Here, we reported on six MS patients treated with NTZ during active SARS-CoV-2 infection.

\section{Cases presentation}

Six patients, including 5 females (83.3\%), with a mean age of $39.2 \pm 9.8$ years, were treated with NTZ while they were infected with SARS-CoV-2. Written informed consent from each reported patient was collected.

At the time of the SARS-CoV-2 infection, all patients were negative to John Cunningham virus (JCV)-IgG and the mean number of NTZ doses administered was $41.2 \pm 24.3$. Four patients received at least one DMT and two patients were treatment naïve before starting NTZ, with only one patient reported clinically relevant comorbidities. Demographical and clinical characteristics of the cohort are summarized in Table 1.

Only two patients reported symptoms suggestive of COVID-19 and underwent nasopharyngeal swab for COVID-19 (Table 2). For the remaining patients, the nasopharyngeal swab was performed for screening purpose before receiving NTZ administration.

All patients were admitted to the University-Hospital G. Rodolico-San Marco's COVID-19 ward, using specific bio-containment measures. NTZ (300 mg) was intravenously administered using the extended dosing protocol [18].

Most of the patients were asymptomatic (7-point ordinal scale for grading clinical status $=1$ ) and were admitted to the hospital for the sole purpose of being administered with NTZ, with the exception of one patient (7-point ordinal scale for grading clinical status $=3$ ), who was hospitalized because of his worsening of the respiratory symptoms. This patient had been previously treated with three DMTs before starting NTZ and reported a previous comorbidity, a mixoid liposarcoma in the right leg, surgically removed in December 2015. The patient did not undergo any chemotherapy.

No patients received O2-therapy or required intensive care. Five of six patients were discharged to homequarantine after 3-4days from the hospitalization and recovered from COVID-19 after about a month (33.5 \pm 10.3 days), with no abnormalities in blood tests. For just one patient, the clinical course was slightly longer (44 days) compared to other patients.

After the hospital discharge, all patients underwent a neurological follow-up visit showing clinically stable condition, with neither relapses nor EDSS worsening. Moreover, no enlarging T2 lesions or new gadoliniumenhanced lesions were observed at the Magnetic Resonance Imaging (MRI) examination performed after recovery from COVID-19.

No respiratory or neurological symptoms have been reported since the hospital discharge. PCR on nasopharyngeal swab was double-negative in all patients. The detection of antibodies against SARS-Cov-2 has not been performed yet.

NTZ was re-administered six weeks later, according to the extended interval dosing, without any complication.

\section{Discussion and conclusions}

This paper reported the clinical outcome of patients with MS receiving NTZ while they were infected by SARS-CoV-2. Currently, there are no data guiding health professionals to manage MS treatments during active SARS-CoV-2 infection. Despite the lack of clinical studies, the EAN, AISM, and ABN recommend to continue all injectable and oral DMTs in patients positive for 
Table 1 Demographical and clinical characteristics of patients

\begin{tabular}{|c|c|c|c|c|c|c|}
\hline Patient & 1 & 2 & 3 & 4 & 5 & 6 \\
\hline Age (years) & 47 & 52 & 39 & 31 & 34 & 32 \\
\hline Sex & female & male & female & female & female & female \\
\hline Disease duration (months) & 203 & 309 & 165 & 105 & 89 & 167 \\
\hline Prior therapies & IFN-beta1b & $\begin{array}{l}\text { IFN-beta1a s.c.; IFN-beta1a i.m.; } \\
\text { cyclophosphamide }\end{array}$ & None & None & fingolimod & IFN-beta1a i.m. \\
\hline $\mathrm{N}$ of relapses at MS diagnosis & 3 & 2 & 2 & 2 & 3 & 2 \\
\hline $\mathrm{N}$ of relapses in the year before starting NTZ & 1 & 2 & 2 & 2 & 1 & 1 \\
\hline $\mathrm{N}$ of relapses during NTZ & 0 & 0 & 0 & 0 & 0 & 0 \\
\hline EDSS at onset & 4.5 & 2.5 & 3.5 & 1.0 & 1.5 & 6.0 \\
\hline EDSS before at NTZ initiation & 5.0 & 2.0 & 3.5 & 0.0 & 1.5 & 4.0 \\
\hline EDSS before COVID infection ${ }^{a}$ & 5.0 & 4.5 & 3.5 & 0.0 & 0.0 & 6.0 \\
\hline $\begin{array}{l}\mathrm{N} \text { of new/enlarged/Gd-enhanced lesions at } \\
\text { MS diagnosis }\end{array}$ & 0 & 0 & 0 & 2 & 3 & 4 \\
\hline $\begin{array}{l}\mathrm{N} \text { of new/enlarged/Gd-enhanced lesions at } \\
\text { NTZ initiation }\end{array}$ & 0 & 0 & 0 & 0 & 0 & 1 \\
\hline $\begin{array}{l}\mathrm{N} \text { of new/enlarged/Gd }+- \text { enhanced lesions } \\
\text { before COVID-19 }\end{array}$ & 0 & 0 & 0 & 0 & 0 & 0 \\
\hline $\begin{array}{l}\mathrm{N} \text { of new/enlarged/Gd }+- \text { enhanced lesions } \\
\text { after COVID-19 }\end{array}$ & 0 & 0 & 0 & 0 & 0 & 0 \\
\hline $\mathrm{N}$ of NTZ administrations before COVID-19 & 28 & 69 & 18 & 13 & 57 & 62 \\
\hline JCV status ${ }^{\mathrm{a}}$ & negative & negative & positive (1.07) & negative & negative & negative \\
\hline Comorbidities & none & myxoid liposarcoma $^{c}$ & none & none & none & none \\
\hline Smoker & no & yes & no & yes & no & no \\
\hline BMI & 21.6 & 25.6 & 24.3 & 27.1 & 21.3 & 25.0 \\
\hline
\end{tabular}

COVID-19: Coronavirus Disease 2019; MS: Multiple Sclerosis; NTZ: natalizumab, IFN: interferon; s.c.: subcutaneously administered; i.m.: intramuscularly administered; EDSS: Expanded Disability Status Scale; Gd: gadolinium; JCV: John Cunningham virus; BMI: body mass index

${ }^{a}$ at last follow-up before COVID-19

b performed after recovery from COVID-19

c surgically treated in December 2015

Table 2 COVID-19 data

\begin{tabular}{|c|c|c|c|c|c|c|}
\hline Patient & 1 & 2 & 3 & 4 & 5 & 6 \\
\hline $\begin{array}{l}\text { Date of the first positive oropharyngeal/ } \\
\text { nasopharyngeal swab }\end{array}$ & $12 / \mathrm{OCT} / 20$ & 14/NOV/20 & 13/JAN/21 & 15/JAN/21 & 16/JAN/21 & 05/FEB/21 \\
\hline Severity of COVID-19 & mild & moderate & mild & mild & mild & mild \\
\hline $\begin{array}{l}\text { 7-point ordinal scale for grading clinical } \\
\text { status of COVID-19 }\end{array}$ & 1 & 3 & 1 & 1 & 2 & 1 \\
\hline COVID-19 treatments & none & $\begin{array}{l}\text { paracetamol, } \\
\text { azitromicine, } \\
\text { prednisolone }\end{array}$ & none & none & paracetamol & none \\
\hline COVID-19 symptoms & none & $\begin{array}{l}\text { fever, sore } \\
\text { throat, loss of } \\
\text { taste, loss of } \\
\text { smell }\end{array}$ & none & none & $\begin{array}{l}\text { headache, bone or joint pain, nasal } \\
\text { congestion, loss of taste, loss of } \\
\text { smell }\end{array}$ & none \\
\hline Hospitalization & no & yes & no & no & no & no \\
\hline $\begin{array}{l}\text { Date of NTZ administration during } \\
\text { COVID-19 }\end{array}$ & $26 / N O V / 20$ & 02/DEC/20 & 14/JAN/21 & 20/JAN/21 & 27/JAN/21 & 10/FEB/21 \\
\hline $\begin{array}{l}\text { Date of the negative oropharyngeal/ } \\
\text { nasopharyngeal swab }\end{array}$ & $30 / \mathrm{NOV} / 20$ & 28/DEC/20 & 28/JAN/21 & 16/FEB/21 & 25/FEB/21 & 08/MAR/21 \\
\hline COVID-19 duration (days) & 39 & 44 & 15 & 32 & 40 & 31 \\
\hline
\end{tabular}

NTZ natalizumab, COVID-19 Coronavirus Disease 2019 
SARS-CoV-2; especially interferons, whose potential antiviral should be also considered [10-12]. According to these recommendations, the concern about a rebound disease activity secondary to NTZ-discontinuation should be kept in mind, individualizing the treatment strategy, such as extended-interval dosing, based on the clinical situation. A recent online survey, promoted by the European Committee for Treatment and Research in Multiple Sclerosis (ECTRIMS), has explored the impact of COVID-19 emergency on MS treatment practices, showing that $70 \%$ of the neurologists reported changes in DMT management. In particular $18-43 \%$ of them would postpone the retreatment with alemtuzumab, cladribine and anti-CD20, while the $64 \%$ would not modify NTZinfusion schedule [19].

Since the COVID-19 has become pandemic, it is common practice in our MS center for all patients to undergo a COVID-19 nasopharyngeal swab test no more than $72 \mathrm{~h}$ prior to the medical service (i.e. follow-up visit, MRI, administration of NTZ dose or other intravenous DMT, etc.). This, together with the possibility to admit patients with confirmed SARS-CoV-2 infection to a COVID-19 ward for their NTZ treatment, allowed to isolate COVID19 cases before coming into contact with other patients.

In our case series, only one patient had a more severe clinical course; however, the contribution of NTZ therapy to his outcome cannot be demonstrated as he had multiple risk factors for severe COVID-19. Particularly, several studies demonstrated that older age is an independent risk factor of severe COVID-19 [5, 6, 19, 20]. In addition, male sex, habit of smoking, and comorbidities (myxoid sarcoma previously surgically treated) may have contributed to the worse clinical course of COVID-19 in this patient. Nonetheless, this patient fully recovered from all COVID-19 symptoms.

In the current literature, both cases of SARS-CoV-2 infection in patients with MS treated with NTZ reported a benign clinical course with complete recovery. Similar to our patients, no worsening of MS clinical conditions was observed, even after the recovery from COVID$19[15,16]$. Particularly, the Authors have suggested that the low viral replication rate, the repeated negative results with PCR for SARS-CoV-2, as well as the benign course of the disease observed in these patients, may be explained by the NTZ mechanism of blockade of integrins, as SARS-CoV-2 may enter human cells through these receptors. According to this theory, the Authors also concluded that the use of drugs that blocking integrins may represent a therapeutic option against SARSCoV-2 infection [15].

Interestingly, recent studies reported a decrease in the total number of natural killer (NK) cells and $\mathrm{CD}^{+} \mathrm{T}$ lymphocytes in patients with COVID-19 [13,
21]. Particularly, the NK cell count reduction was more marked in patients with more severe COVID-19 disease compared to milder cases. Since NTZ induce an increase in circulating NK cells by reducing their migration into the CNS, it also may potentially represent an alternative treatment for COVID-19 [22, 23].

It should be also noted that one fatal case of COVID-19 was described in a 51-year-old African American woman with MS on NTZ treatment. However, because of the presence of multiple risk factors for severe COVID-19 (race, obesity, hypertension, and elevated inflammatory markers), it is unclear whether NTZ may have played a role on it [24]. In addition, several concerns have been raised about the effect of NTZ in reducing the lymphocytes migration to the lungs and mucosa that may potentially increase the viral shedding [25]. NTZ also reduces immune surveillance in the CNS and, thus, as shown for PML, may potentially increase the risk of COVID-19 encephalitis [25].

Notwithstanding these concerns, a recent Italian multicenter study of the Musc-19 group confirmed that NTZ has shown an acceptable safety profile during the COVID-19 pandemic, with no significant increased risk of severe COVID-19 course, even after adjusting for several confounders [6].

Another study recently investigated self-reported COVID-19 symptoms and disease severity in MS patients under NTZ or fingolimod with serology confirmation for SARS-CoV-2 infection. In this study $13.4 \%$ patients on NTZ or fingolimod had anti-SARS-CoV-2 antibodies, showing no or only mild COVID-19 symptoms and, thus, supporting the hypothesis that it is safe to continue treatment with these drugs in the current setting [26].

Furthermore, in the light of the recent development of new SARS-CoV-2 viral protein and inactivated vaccines, it is also conceivable that NTZ may induce an adequate response to the COVID-19 immunization, as suggested from several influenza vaccine studies [27, 28].

In conclusion, MS is a chronic and severe disease with high risk of disability accumulation especially in patients with more aggressive course, who require more aggressive treatment approach from the beginning of the therapeutic management [29]. Particularly, NTZ has proved highly effective, both in clinical trials and in real-word setting, reducing the rate of clinical relapses and slowing the disease progression [30]. However, during the COVID-19 pandemic, it was observed that NTZ administrations have been discontinued or delayed because of fear of more severe course of COVID-19 [19].

Our cases series may encourage Clinicians to not discontinue or delay NTZ administration and/or to not switch to different DMT in selected patients with active SARS-CoV-2 infection. Thus, it could be suggested to 
continue NTZ therapy per protocol in COVID-19 wards, using specific bio-containment measures.

Despite the fears associated with the admission to the COVID-19 ward, in our cases study all patients accepted to be hospitalized and were dismissed after about 3-4 days.

In conclusion, although the limit of the small sample size, our single-center experience shows that treatment with NTZ during pandemic is relatively safe and could be continued in selected patients who are infected by SARS$\mathrm{CoV}-2$, thereby reducing the risk of MS rebound commonly reported after NTZ discontinuation.

\begin{abstract}
Abbreviations
ABN: Association of British Neurologists; AISM: Associazione Italiana Sclerosi Multipla; COVID 19: Coronavirus Disease 19; DMT: Disease-modifying therapy; EAN: European Academy of Neurology; JCV: John Cunningham virus; MS: Multiple Sclerosis; NK: Natural killer; NTZ: Natalizumab; PML: Progressive Multifocal Leukoencephalopathy; SARS-CoV-2: Severe Acute Respiratory Syndrome Coronavirus 2.
\end{abstract}

\section{Acknowledgements}

The authors extend their appreciation to these patients for their participation.

\section{Authors' contributions}

CGC contributed to the study plan, handled database, interpreted the data, drafted and approved the manuscript. ST, CF, SA handled database, interpreted the data and approved the manuscript. AM interpreted the results and approved the final manuscript. FP interpreted data, discussed the results, approved the final manuscript and took the responsibility for the submission. All authors have read and approved the manuscript.

\section{Funding}

The study was not funded.

\section{Availability of data and materials}

Dataset is available under reasonable request.

\section{Declarations}

Ethics approval and consent to participate

This study complies with the guidelines for human studies and was conducted ethically in accordance with the World Medical Association Declaration of Helsinki. Written informed consent was obtained from all participants or their legal surrogate. The Ethics Committee (Catania 1) of University of Catania approved this study.

\section{Consent for publication}

All the participants or their legal surrogate gave written consent for their personal or clinical details to be published in this study.

\section{Competing interests}

The author(s) declared the following potential conflicts of interest with respect to the research, authorship, and/or publication of this article: Clara G Chisari received a research grant from Sanofi and grant for congress participation from Almiral, Biogen, Merck Serono, Novartis, Roche, Sanofi and TEVA; Simona Toscano declares no conflict of interest; Sebastiano Arena declares no conflict of interest; Chiara Finocchiaro declares no conflict of interest; Arturo Montineri declares no conflict of interest; Francesco Patti has received honoraria for speaking activities by Bayer Schering, Biogen, Merck Serono, Novartis and Sanofi Aventis; he also served as advisory board member the following companies: Bayer Schering, Biogen, Merck Serono, Novartis; he was also funded by Pfizer and FISM for epidemiological studies; he received grant for congress participation from Bayer Schering, Biogen Idec, Merck Serono, Novartis, Roche, Sanofi Aventis and TEVA.

\section{Author details}

${ }^{1}$ Department of Medical and Surgical Sciences and Advanced Technologies "G.F. Ingrassia", section of Neurosciences, University of Catania, via Santa Sofia 78, 95123 Catania, Italy. ${ }^{2}$ Infectious Diseases and Tropical Unit, Azienda Ospedaliero Universitaria "Policlinico-San Marco", via Santa Sofia 78, 95123 Catania, Italy.

Received: 27 July 2021 Accepted: 29 September 2021

Published online: 29 November 2021

\section{References}

1. Zhou L, Zhang M, Wang J, Gao J. Sars-Cov-2: underestimated damage to nervous system. Travel Med Infect Dis. 2020;36:101642.

2. Li J, Huang DQ, Zou B, Yang H, Hui WZ, Rui F, et al. Epidemiology of COVID-19: a systematic review and meta-analysis of clinical characteristics, risk factors, and outcomes. J Med Virol. 2021;93(3):1449-58.

3. Zabalza A, Cardenas-Robledo S, Tagliani P, Arrambide G, Otero-Romero S, Carbonell-Mirabent P, et al. COVID-19 in multiple sclerosis patients: susceptibility, severity risk factors and serological response. Eur J Neurol. 2020;28(10):3384-95. Epub 2021 Jan 9.

4. Chaudhry F, Bulka H, Rathnam AS, Said OM, Lin J, Lorigan H, et al. COVID19 in multiple sclerosis patients and risk factors for severe infection. J Neurol Sci. 2020;418:117147.

5. Louapre C, Collongues N, Stankoff B, Giannesini C, Papeix C, Bensa C, et al. Clinical characteristics and outcomes in patients with coronavirus disease 2019 and multiple sclerosis. JAMA Neurol. 2020;77(9):1079-88.

6. Sormani MP, De Rossi N, Schiavetti I, Carmisciano L, Cordioli C, Moiola L, et al. Disease-modifying therapies and coronavirus disease 2019 severity in multiple sclerosis. Ann Neurol. 2021:89(4):780-9.

7. Zheng C, Kar I, Chen CK, Sau C, Woodson S, Serra A, et al. Multiple sclerosis disease-modifying therapy and the COVID-19 pandemic: implications on the risk of infection and future vaccination. CNS Drugs. 2020;34(9):879-96.

8. Hartung HP, Aktas O. COVID-19 and management of neuroimmunological disorders. Nat Rev Neurol. 2020;16(7):347-8.

9. Thakolwiboon S, Zhao-Fleming H, Pan J, Scott JK, Shoji E, Sohn G, et al. Disease-modifying therapies during the COVID-19 outbreak: a narrative review of international and National Recommendations. Int J MS Care. 2020;22(4):151-7.

10. European Multiple Sclerosis Platform. Updated global COVID-19 advice for people with MS. https://emsp.org/news/coronavirus-disease-covid19-and-multiple-sclerosis.

11. Associazione Italiana Sclerosi Multipla (AISM): Guidance for the management of the patients with MS during COVID-19 pandemic. https://www. aism.it/sites/default/files/ComunicazioneGdSSINSM-Coronavirus.pdf 2021. Accessed 1 Sept 2021.

12. MS Society: COVID-19 coronavirus and MS treatments. https://www. mssociety.org.uk/about-ms/treatments-and-therapies/disease-modif ying-therapies/covid-19-coronavirus-and-ms. Accessed 1 Sept 2021.

13. Zheng M, Gao Y, Wang G, Song G, Liu S, Sun D, et al. Functional exhaustion of antiviral lymphocytes in COVID-19 patients. Cell Mol Immunol. 2020;17(5):533-5.

14. Clerico M, Artusi CA, Liberto AD, Rolla S, Bardina V, Barbero P, et al. Natalizumab in multiple sclerosis: long-term management. Int J Mol Sci . 2017:18(5):940

15. Aguirre C, Meca-Lallana V, Barrios-Blandino A, Del Rio B, Vivancos J. Covid19 in a patient with multiple sclerosis treated with natalizumab: may the blockade of integrins have a protective role? Mult Scler Relat Disord. 2020:44:102250.

16. Borriello G, lanniello A. COVID-19 occurring during Natalizumab treatment: a case report in a patient with extended interval dosing approach. Mult Scler Relat Disord. 2020;41:102165.

17. Brownlee W, Bourdette D, Broadley S, Killestein J, Ciccarelli O. Treating multiple sclerosis and neuromyelitis optica spectrum disorder during the COVID-19 pandemic. Neurology. 2020;94(22):949-52.

18. Chisari CG, Grimaldi LM, Salemi G, Ragonese P, laffaldano P, Bonavita $\mathrm{S}$, et al. Clinical effectiveness of different natalizumab interval dosing schedules in a large Italian population of patients with multiple sclerosis. J Neurol Neurosurg Psychiatry. 2020;91(12):1297-303. 
19. Portaccio E, Fonderico M, Hemmer B, Derfuss T, Stankoff B, Selmaj K, et al. Impact of COVID-19 on multiple sclerosis care and management: results from the European Committee for Treatment and Research in Multiple Sclerosis survey. Mult Scler. 2021:1352458521 1005339. https://doi.org/10. 1177/13524585211005339. Epub ahead of print.

20. Sormani MP, Salvetti M, Labauge P, Schiavetti I, Zephir H, Carmisciano L, et al. DMTs and Covid-19 severity in MS: a pooled analysis from Italy and France. Ann Clin TransI Neurol. 2021;8(8):1738-44.

21. Hadjadj J, Yatim N, Barnabei L, Corneau A, Boussier J, Smith N, et al. Impaired type I interferon activity and inflammatory responses in severe COVID-19 patients. Science. 2020;369(6504):718-24.

22. Kaufmann M, Haase R, Proschmann U, Ziemssen T, Akgun K. Real-world lab data in Natalizumab treated multiple sclerosis patients up to 6 years long-term follow up. Front Neurol. 2018;9:1071.

23. Al-Ani M, Elemam NM, Hundt JE, Maghazachi AA. Drugs for multiple sclerosis activate natural killer cells: do they protect against COVID-19 infection? Infect Drug Resist. 2020;13:3243-54.

24. Rimmer K, Farber R, Thakur K, Braverman G, Podolsky D, Sutherland L, et al. Fatal COVID-19 in an MS patient on natalizumab: a case report. Mult Scler J Exp TransI Clin. 2020;6(3):2055217320942931.

25. Giovannoni G, Hawkes C, Lechner-Scott J, Levy M, Waubant E, Gold J. The COVID-19 pandemic and the use of MS disease-modifying therapies. Mult Scler Relat Disord. 2020;39:102073.

26. Mallucci G, Zito A, Baldanti F, Gastaldi M, Fabbro BD, Franciotta D, et al. Safety of disease-modifying treatments in SARS-CoV-2 antibody-positive multiple sclerosis patients. Mult Scler Relat Disord. 2021;49:102754.

27. Metze C, Winkelmann A, Loebermann M, Hecker M, Schweiger B, Reisinger EC, et al. Immunogenicity and predictors of response to a single dose trivalent seasonal influenza vaccine in multiple sclerosis patients receiving disease-modifying therapies. CNS Neurosci Ther. 2019:25(2):245-54.

28. Ciotti JR, Valtcheva MV, Cross AH. Effects of MS disease-modifying therapies on responses to vaccinations: a review. Mult Scler Relat Disord. 2020:45:102439.

29. Montalban X, Gold R, Thompson AJ, Otero-Romero S, Amato MP, Chandraratna $\mathrm{D}$, et al. ECTRIMS/EAN guideline on the pharmacological treatment of people with multiple sclerosis. Eur J Neurol. 2018;25(2):215-37.

30. Wiendl H, Spelman T, Butzkueven H, Kappos L, Trojano M, Su R, et al. Real-world disability improvement in patients with relapsing-remitting multiple sclerosis treated with natalizumab in the Tysabri observational program. Mult Scler. 2021;27(5):719-28.

\section{Publisher's Note}

Springer Nature remains neutral with regard to jurisdictional claims in published maps and institutional affiliations.

Ready to submit your research? Choose BMC and benefit from:

- fast, convenient online submission

- thorough peer review by experienced researchers in your field

- rapid publication on acceptance

- support for research data, including large and complex data types

- gold Open Access which fosters wider collaboration and increased citations

- maximum visibility for your research: over 100M website views per year

At BMC, research is always in progress.

Learn more biomedcentral.com/submissions 